\title{
Aktuelle Anforderungen der Rechtsprechung an die Belehrung gegenüber Wahlleistungspatienten
}

\section{Einführung \\ $\nabla$}

Seitdem zunehmend auch die privaten Krankenversicherungen bei der Kostenerstattung Kürzungen vornehmen und Patienten ihre Erstattungsansprüche ganz oder in Teilen zunehmend nicht mehr realisieren können, sind Honoraransprüche aufgrund von Wahlleistungsvereinbarungen vermehrt Gegenstand gerichtlicher Auseinandersetzungen geworden. In $2 \mathrm{ak}$ tuellen Entscheidungen haben das Landgericht Stuttgart und der Bundesgerichtshof (BGH) die formellen Anforderungen an Wahlleistungsvereinbarungen näher konkretisiert. Die Entscheidungen haben weitreichende Konsequenzen für die Praxis.

\section{Neue Anforderungen an den Inhalt der Wahlleistungsvereinbarung $\nabla$}

Das Landgericht Stuttgart befasste sich in seinem Urteil vom 04.05.2016, Az. 13 S 123/15, mit der Honorarklage eines liquidationsberechtigten Chefarztes, dessen Patientin die Wahlleistung „Chefarztbehandlung" mit ihm vereinbart hatte. Die Wahlleistungsvereinbarung enthielt unter anderem die folgende Formulierung:

„Ausdrücklich wird nochmals darauf hingewiesen, dass sich die Vereinbarung über zusätzliche wahlärztliche Leistungen auf alle an der Behandlung beteiligten Ärztlichen Direktoren/Ärzte, soweit diese zur Erbringung wahlärztlicher Leistungen berechtigt sind, einschließlich der von diesen Ärzten veranlassten Leistungen durch Ärzte und ärztlich gerichtete Einrichtungen außerhalb des Klinikums erstreckt („Wahlarztkette nach $\S 17$ Abs. 3 KHEntgG)“.

Daneben erhielt die Patientin eine Patienteninformation mit einer Liste der angestellten und beamteten Ärzte des Krankenhauses, deren Erhalt sie bestätigte. Als bekannt wurde, dass der später klagende Chefarzt am Tag der geplanten Behandlung verhindert sein würde, informierte er die Patientin und zeigte ihr verschiedene Alternativen auf, die Operation zu verschieben, sie als allgemeine Krankenhaus- leistung durch den diensthabenden Arzt durchführen zu lassen oder sich von seinem namentlich benannten Vertreter behandeln zu lassen. Die Beklagte entschied sich für die Operation durch den Stellvertreter des Chefarztes und unterzeichnete eine Stellvertretervereinbarung. Nachdem die Patientin die Rechnung des Chefarztes nicht beglich, machte dieser seinen Honoraranspruch gerichtlich geltend. Das Amtsgericht hatte der Klage in 1. Instanz stattgegeben. Das Landgericht Stuttgart hob das Urteil in der Berufungsinstanz jedoch auf und wies die Klage des Chefarztes mit der Begründung ab, dass die Wahlleistungsvereinbarung unwirksam sei. Die zwischen Krankenhaus und dem Patienten geschlossene Vereinbarung beschränke sich, so das Gericht, nicht darauf, das wiederzugeben, was ohnehin Inhalt des $§ 17$ Abs. 3 Satz 1 KHEntG ist.

\section{$\S 17$ Abs. 3 Satz 1 KHEntgG lautet:} „Eine Vereinbarung über wahlärztliche Leistungen erstreckt sich auf alle an der Behandlung des Patienten beteiligten angestellten oder beamteten Ärzte des Krankenhauses, soweit diese zur gesonderten Berechnung ihrer Leistungen im Rahmen der vollstationären und teilstationären sowie einer vor- und nachstationären Behandlung ( $\$ 115 a$ des Fünften Buches Sozialgesetzbuch) berechtigt sind, einschließlich der von diesen Ärzten veranlassten Leistungen von Ärzten und ärztlich geleiteten Einrichtungen außerhalb des Krankenhauses; darauf ist in der Vereinbarung hinzuweisen."

Zweck der Vorschrift ist aber, den Verwender einer Wahlleistungsklausel dazu zu verpflichten, potentielle Patienten auf den Inhalt der gesetzlichen Vorschrift hinzuweisen. Die streitige Klausel habe jedoch den in $\S 17$ Abs. 3 Satz 1 KHEntG genannten Kreis der liquidationsberechtigten Ärzte unzulässig erweitert. Dieser erstreckt die „Wahlarztkette“ ausschließlich auf „angestellte oder beamtete“ Ärzte. Im zugrundeliegenden Fall habe die konkrete Formulierung diesen Kreis etwa auf Honorar-, Beleg- oder Konsiliarärzte erweitert. Allein durch die Verwendung des Wortes „Wahlarztkette“ im Klammerzu-

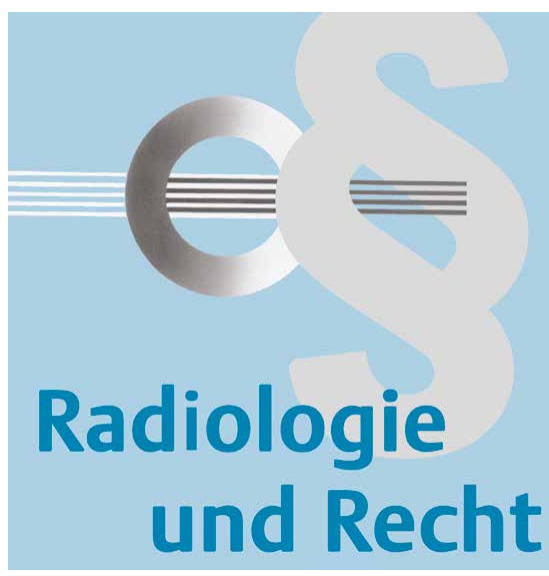

satz hinter der streitigen Formulierung werde diese unzulässige Erweiterung nicht geheilt. Das Argument des Chefarztes, durch die im Klammerzusatz festgehaltene Bezugnahme auf die Wahlarztkette des $\S 17$ Abs. 3 KHEntG sei ausreichend, um der vom Gesetz geforderten Hinweisfunktion zu genügen, ließ das Gericht nicht gelten. Denn einerseits werde nur auf die Wahlarztkette und damit auf den verkürzten zweiten Teil der Norm Bezug genommen und nicht auf den verkürzten ersten Teil. Andererseits werde durch die streitbefangene Formulierung gerade eine solche gewählt, die dem Gesetzeswortlaut nicht entspricht. Die Wahlleistungsvereinbarung mache also deutlich, dass sie vom Gesetzeswortlaut abweichen wolle. Dies könne nicht durch Bezugnahme auf eine Norm, welche nicht denselben Inhalt wie die Vereinbarung hat, abgeändert werden.

Der Hinweis auf die Patienteninformation, die die angestellten und beamteten Ärzte aufführt und an dieser Stelle auch $\S 17$ Abs. 3 Satz 1 KHEntgG vollständig wiedergibt, ändert nach Auffassung des Landgerichts Stuttgart nichts an der Beurteilung. Die Patienteninformationen seien nicht Bestandteil der Vereinbarung, sondern erläuterten diese lediglich. Dementsprechend würden die Patienteninformationen nicht unterzeichnet, sondern es werde lediglich deren Erhalt bestätigt. Nachdem $§ 17$ Abs. 2 KHEntgG die Schriftform für die Wahlleistungsvereinbarung und die hierfür zu erhebenden Entgelte vorschreibt, komme dem genauen Wortlaut der Wahlleistungsvereinbarung eine entscheidende Bedeutung zu. Rechtsfolge dieses formellen Fehlers sei die Unwirksamkeit der Wahlleistungsvereinbarung. Dabei verwies das Landgericht Stuttgart auf eine Entscheidung des BGH vom 16.10.2015 (Az.: III ZR 85/14), wonach §17 
Abs. 3 Satz 1 KHEntgG ein Verbotsgesetz darstelle, da der Kreis der liquidationsberechtigten Wahlärzte in dieser Norm abschließend festgelegt werde. Der BGH hat hierzu ausgeführt, es handele sich bei § 17 Abs. 3 KHEntgG um eine dem Schutz des Privatpatienten dienende, zwingende preisrechtliche Norm. Eine Wahlleistungsvereinbarung, durch welche versucht wird, diesen Kreis zu erweitern, verstoße gegen diese gesetzlichen Vorgaben und sei deswegen nichtig.

Daraus folge nach Ansicht des Landgerichts Stuttgart die Gesamtnichtigkeit der Wahlleistungsvereinbarung und des gesamten Arztvertrags, weil der Arztvertrag ohne die Wahlleistungsvereinbarung keinen Bestand haben könne. Nach der Rechtsprechung des BGH sei dies selbst bei äußerlich eigenständigen Verträgen der Fall, wenn nach den Interessen der Vertragspartner unter Berücksichtigung der Verkehrssitte die Verträge derart verknüpft seien, dass ihre Wirksamkeit voneinander abhingen. Der Inhalt der Wahlleistungsvereinbarungen werde im Regelfall durch den Krankenhausträger vorgegeben; dies ähnele rechtsgedanklich den Fällen, die Allgemeine Geschäftsbedingungen betreffen. Die Wahlleistungsvereinbarung werde als Zusatzvereinbarung Vertragsbestandteil des Arztvertrags. Grundsätzlich würden zwar gemäß §306 Abs. 2 BGB statt des unwirksamen Vertragsbestandteils die gesetzlichen Vorschriften gelten, sodass der restliche Vertrag bestehen bleiben würde. Das Landgericht Stuttgart wandte jedoch die ständige Rechtsprechung des BGH zum Verbot der geltungserhaltenden Reduktion auf den Fall an. Demnach dürfen unzulässige Allgemeine Geschäftsbedingungen im Falle ihrer Unwirksamkeit nicht auf den gerade noch zulässigen Teil reduziert werden. Dies würde den Verwendern (hier den Krankenhausträgern) einen Anreiz liefern, ihre Klauseln mit einseitig begünstigendem Inhalt zu formulieren. So könnten sie abwarten, ob dem Vertragspartner dieser Fehler auffällt und würden im schlimmsten Fall wegen der Geltungsreduktion auf den noch zulässigen Inhalt weiterhin einen Anspruch auf die Gegenleistung (in diesem Fall die Vergütung) zu den Bedingungen behalten, zu denen sie ohnehin den Vertrag hätten abschließen müssen. Um dem entgegen $\mathrm{zu}$ wirken, verbietet der BGH eine solche Geltungsreduktion. Diese Rechtsauffassung übertrug das Landgericht Stuttgart auf den vorliegenden Fall, sodass die mangelhafte Wahl- leistungsklausel zur Unwirksamkeit des gesamten Arztvertrages führte, was wiederum zur Rechtsfolge hatte, dass der Chefarzt gegenüber dem Patienten keinen Vergütungsanspruch hatte. Dem Krankenhaus verblieben somit letztlich nur die üblichen Pflegesätze.

\section{Rechtliche Konsequenzen des Verstoßes gegen Aufklärungs- pflichten bei Wahlleistungs- vereinbarungen \\ $\nabla$}

Am 19.07.2016 entschied der BGH (Az. VI ZR 75/15) über den Schmerzensgeldanspruch eines Patienten, der ohne sein Wissen von einem stellvertretenden Oberarzt operiert wurde, nachdem er mit dem beklagten Krankenhaus die Wahlleistung Chefarztbehandlung bezüglich einer stationären chirurgischen Handoperation abgeschlossen hatte. In die Operation durch den stellvertretenden Oberarzt hatte der Patient nicht eingewilligt und wurde über die Verhinderung des Chefarztes nicht informiert. Postoperativ stellten sich bei dem Patienten an der operierten Hand erhebliche gesundheitliche Beeinträchtigungen ein. Das Krankenhaus erhob den Einwand rechtmäßigen Alternativverhaltens, da der Eingriff in seiner konkreten Ausführung nicht anders verlaufen wäre, wenn ihn der Chefarzt vorgenommen hätte. Ein gerichtlich bestellter Sachverständiger stellte fest, dass der stellvertretende Oberarzt fehlerfrei operiert hatte. Nach Auffassung des Krankenhauses stand der Kläger daher genauso da, als wenn die Operation, wie von ihm erwartet und konsentiert, vom Chefarzt vorgenommen worden wäre. Die Vorinstanzen wiesen die Klage ab, der BGH hob auf die Revision des Patienten das Urteil des Oberlandesgerichts Koblenz auf.

Nach Ansicht des BGH lag keine wirksame Einwilligung des Patienten in die Operation vor, die durch den stellvertretenden Oberarzt durchgeführt wurde. Bei einem Chefarztvertrag sei die persönliche Vornahme des Eingriffs wesentlicher Vertragsbestandteil. Schließlich zahle der Patient bei einer solchen Vereinbarung mehr als der Kassenpatient, der sich den behandelnden Arzt nicht aussuchen könne. Demnach ging der Patient von einer Operation des Chefarztes aus und wollte lediglich in diese Behandlung einwilligen. Vor diesem Hintergrund ergäben sich für eine hypothetische Einwilligung in eine Oberarztbehandlung keine Anhaltspunk- te, so der BGH. In den Vorinstanzen entschieden die Gerichte, dem Schmerzensgeldanspruch des Klägers stehe die Figur des rechtmäßigen Alternativverhaltens entgegen, da die postoperativen Folgen auch bei einer Operation durch den Chefarzt hätten entstehen können, da sie nicht Folge einer fehlerhaften Operation seien. Der BGH wies aber auf seine ständige Rechtsprechung hin, nach der der Schutzzweck der jeweiligen Norm entscheide, wie erheblich dieser Einwand sei. Der BGH entschied, dass der Schutzzweck des Einwilligungserfordernisses nicht mit dem Alternativverhalten zu vereinbaren sei. Durch die Voraussetzung einer wirksamen Einwilligung werde das Recht, über den eigenen Körper zu bestimmen, durch die Menschenwürde und das Recht auf körperliche Unversehrtheit grundgesetzlich geschützt. Es handele sich um ein absolutes Recht, das für den medizinischen Eingriff nur in dem konkreten Umfang und unter den festgelegten Umständen aufgehoben werde. Aus diesem Grund hätte es einer schriftlichen Stellvertretervereinbarung bedurft. Der Eingriff selbst stelle eine Verletzung der körperlichen Integrität dar und das Vertrauen, das aufgrund der Vereinbarung und der Einwilligung bezüglich einer Chefarztbehandlung bestand, müsse geschützt werden.

In dem Urteil wird die Diskussion um die Frage, ob das Vorliegen eines Schadens lediglich aus dem Verstoß gegen das Selbstbestimmungsrecht ableitbar ist, aufgegriffen. In dem angesprochenen Urteil des BGH vom 27.05.2008 (Az.: VI ZR 69/07) wurde eine Haftung nur aus einer Verletzung des Persönlichkeitsrechts auch ohne eine daraus resultierende Schädigung durch den Arzt verneint. Zwar sei die Handlung mangels einer Einwilligung rechtswidrig gewesen, aber es sei dadurch kein Schaden entstanden, der ersetzt werden könne. Damit hob der BGH das Urteil des Oberlandesgerichts Thüringen vom 03.12.1997 (Az.: 4 U 687/97) auf, nach dessen Auffassung die Verletzung des Selbstbestimmungsrechtes allein eine Haftung begründe, obwohl der Kausalverlauf auch ohne Pflichtverletzung gleichgeblieben wäre. In dem vorliegenden Urteil war der BGH der Ansicht, dass seine Entscheidung dem Urteil von 2008 nicht entgegenstünde. Er sieht in dem enttäuschten Vertrauen und dem Eingriff ohne Einwilligung eine ausreichende Grundlage für einen immateriellen Schmerzensgeldanspruch. 


\section{Strafrechtliche Konsequenz einer fehlenden Aufklärung \\ $\nabla$}

Bei einer fehlenden Aufklärung über die Verhinderung des Chefarztes entfällt nicht nur der Honoraranspruch des liquidationsberechtigten Chefarztes bzw. des Krankenhauses. Der Arzt, der anstelle des Chefarztes operiert, ohne dass der Patient informiert und eine Stellvertretervereinbarung geschlossen wurde, setzt sich auch dem Vorwurf einer vorsätzlichen Körperverletzung gemäß §223 StGB aus. Darüber hinaus muss der Chefarzt, der um seine Verhinderung weiß und den Patienten nicht hierüber aufklärt, sondern seinen Stellvertreter ohne entsprechende Vereinbarung mit dem Patienten operieren lässt, um diese Leistungen als eigene abrechnet, mit einem Ermittlungsverfahren wegen (versuchten) Abrechnungsbetruges rechnen.

\section{Ergebnis}

Zur Vermeidung dieser rechtlichen Konsequenzen sollten Radiologen größten Wert auf den Abschluss einer wirksamen Wahlleistungsvereinbarung und die ordnungsgemäße Aufklärung des Patienten legen. Eine wirksame Einwilligung setzt die vorherige, ordnungsgemäße Aufklärung des Patienten voraus, da andernfalls sein Selbstbestimmungsrecht verletzt würde. Das Selbstbestimmungsrecht leiten die Gerichte aus dem Schutz der Menschenwürde und dem Recht auf körperliche Unversehrtheit (Art. 1 Abs. 1, Art. 2 Abs. 1 und 2 GG) her. Selbst ein geringer Aufklärungsfehler führt daher nach Ansicht des BGH zur Gesamtunwirksamkeit der Einwilligung (Urteil vom 13.01.1987, Az.: VI ZR 65/88). Aus einem solchen Fehler und der Verletzung des Selbstbestimmungsrechts folgt eine rechtswidrige Handlung, die eine deliktische Haftung nach sich zieht und überdies strafbar ist. Hätte das Argument des rechtmäßigen Alternativverhaltens Anwendung gefunden und eine Haftung ausgeschlossen, wäre das Selbstbestimmungsrecht faktisch ausgehebelt worden. Chef- und Oberärzte, die in einem Krankenhaus Wahlleistungen erbringen, sollten daher eine rechtssichere Wahlleistungsvereinbarung verwenden, im Verhinderungsfalle den Patienten frühzeitig informieren sowie eine schriftliche Stellvertretervereinbarung mit ihm abschließen (eingehend zu den konkreten Anforderungen an derartige Vereinbarungen und zum korrekten Zeitpunkt der Aufklärung und des Abschlusses Wigge / Flau, RöFö 9/2016, S. 882 bis 884).

\section{René T. Steinhäuser}

Rechtsanwalt

Ulrike Urbaneck

Rechtsanwältin

Rechtsanwälte Wigge

Neuer Wall 44

20354 Hamburg

Telefon: (040) 3398705-90

Telefax: (040) 3398705-99

Internet: www.ra-wigge.de

E-Mail: kanzlei@ra-wigge.de 